\title{
FATIGUE BEHAVIOR IN MONOCRYSTALLINE NI-BASED SUPERALLOYS FOR BLADE APPLICATIONS
}

\author{
C.A. Yablinsky ${ }^{1}$, K.M. Flores ${ }^{1}$, M.J. Mills ${ }^{1}$, J.C. Williams ${ }^{1}$, Joe Rigney ${ }^{2}$ \\ ${ }^{1}$ The Ohio State University; 477 Watts Hall, 2041 College Road; Columbus, OH 43210, USA \\ ${ }^{2}$ GE Aviation; 1 Neumann Way; Cincinnati, OH 45215, USA
}

Keywords: sustained peak low cycle fatigue, creep-fatigue, deformation mechanisms

\begin{abstract}
Ni-based superalloys are used for turbine airfoil applications due to their excellent high temperature properties. Alloy design has historically focused on creep resistance as the critical design limiting parameter. Recently, the focus has shifted to include fatigue resistance, resulting in the need to better understand the effects of alloy microstructure on fatigue crack initiation and propagation.
\end{abstract}

In this study, sustained peak low cycle fatigue tests of coated monocrystalline René N5 specimens oriented with the loading axis along [001] were conducted in strain control in the temperature range of $980-1100^{\circ} \mathrm{C}$. Tests were performed with either a tensile dwell (hold) time or a compressive dwell time. Fracture surfaces were characterized via scanning electron microscopy (SEM). Microstructural analysis was performed using a high resolution SEM. Far field deformation mechanisms were examined perpendicular to the loading axis and parallel to the loading axis using transmission electron microscopy. The occurrence of creep-fatigue interaction was characterized by the different types of rafting seen, and the deformation mechanisms varied between specimens with compressive hold time and tensile hold time.

This project is a collaborative effort with GE Aviation and is funded by the AFOSR under the MEANS2 program.

\section{Introduction}

Nickel based superalloys have been used historically in the aerospace industry for turbine airfoil applications. The alloys are useful at high temperatures because of their creep resistance, along with hot corrosion and oxidation resistance [1,2]. Until recently, creep has been the limiting factor for turbine airfoils, and thus creep mechanisms have been the subject of numerous studies [1-6]. Early generation alloying of monocrystalline alloys was developed to improve creep resistance by adding slower diffusing elements, such as rhenium and ruthenium. Rhenium additions improve creep rupture life and oxidation resistance [7,8], while ruthenium decreases overall density compared to rhenium, stabilizes the microstructure, and increases oxidation resistance $[8,9]$. Additionally, creep resistance increases with a decreasing number of grain boundaries, and therefore the invention of the monocrystalline blade increased creep resistance. Coincidentally, fatigue resistance has tended to increase with improvements in creep resistance. However, more recently fatigue failures have been experienced. Consequently, fatigue mechanisms have become of interest.
It is known that engine efficiency increases with increasing temperature, and therefore the need for alloys that can withstand higher temperatures is necessary. Past engine designs have been limited by the melting temperature of the blade material, and ceramic systems are not feasible due to fracture issues. However, more recent changes in modern high pressure turbine (HPT) blade designs incorporate cooling channels, which allow an increase in the engine operating temperature while keeping the blade at an acceptable maximum temperature. The introduction of cooling channels has caused local stresses, especially within the thin regions between the channels, and temperature gradients across the blade to increase. In these HPT components, experience and experimental studies have shown that fatigue can be a life-limiting factor. The changing limitation from creep to fatigue has highlighted the need for a comprehensive study of fatigue deformation mechanisms, as well as creep-fatigue mechanisms, since the engine operates at high temperature where creep occurs.

Previously, creep-fatigue studies have observed that cyclic creep decreases lifetime compared to creep alone [10]. A study by Zrnik observed fracture surfaces and dislocation structures in specimens that were subjected to static loading, cyclic loading and cyclic loading with a hold at maximum load. Static tests were performed in tension while the cyclic tests were tension-tension, with an R-ratio $\left(\mathrm{P}_{\min } / \mathrm{P}_{\max }\right)$ of 0.0125 . After static loading, tangles of dislocations were present in both the $\gamma$ and $\gamma^{\prime}$ phases and the fracture surface was indicative of pure creep. Under cyclic loading conditions, as the hold time decreased to 0 (purely cyclic loading), the dislocations became more confined to the matrix phase alone. The fracture surfaces of specimens with longer hold times were similar to specimens failed in pure creep, while specimens with shorter hold times had fatigue striations, like the fracture surfaces seen in the pure fatigue case [11]. While these observations provide insight into the transition from creep to creep-fatigue to fatigue deformation, the results are only representative of the tension case. Furthermore, no observations of the microstructural changes with deformation were noted.

The object of this study is to characterize the sustained peak low cycle fatigue behavior of Rene N5, a second-generation single crystal nickel based superalloy containing 3\% rhenium. Tests with tension and compression dwell were performed. We observe that fracture modes and microstructural evolution differ between compression and tension dwell. The deformation character for each of the test types was found to be dislocation network formation and $\gamma^{\prime}$ shearing, respectively. Creep-fatigue interactions influence the deformation behavior more than either creep or fatigue alone. 


\section{Experimental Procedure}

For this study, the material of focus was monocrystalline nickelbased superalloy René N5. Specimens were prepared by GE Aviation and tested by Metcut (Cincinnati, OH). Coated, cylindrical specimens oriented with the [001] axis parallel to the loading direction were tested in air at $980-1100^{\circ} \mathrm{C}(1800$ $2000^{\circ} \mathrm{F}$ ). Sustained peak low cycle fatigue (SPLCF) tests were performed in total strain control, with a total strain range between 0.4 and 1.2 percent, and a 2 minute dwell (hold) time in either compression or tension, as illustrated in the strain vs. time schematics in Figures 1 and 2, respectively. The corresponding stress vs. time behavior is also shown schematically. During each cycle, the material yields during loading to peak strain. During the hold time, stress relaxation occurs which coincides with creep in the material. Over the course of the compression hold experiment, the mean stress increases and a positive mean stress develops by mid-life, while in the tension hold experiment, the mean stress decreases but stays positive. The peak stress stabilized after about $10-20 \%$ of the total life, and was less than $350 \mathrm{MPa}$ for all specimens. All specimens failed under 20,000 cycles. The fractured specimens were provided to OSU for analysis.

Fracture surfaces were characterized using an FEI Quanta scanning electron microscope (SEM). The fracture surfaces were characterized parallel to loading axis. The specimens were then sectioned for both SEM and transmission electron microscopy (TEM) analysis. Microstructures and substructures were investigated parallel and perpendicular to the loading axis, having normal directions of [100] and [001] respectively. Samples with a [100] normal were oriented using orientation image mapping techniques. Specimens for SEM were mounted in conductive Bakelite and etched with a solution of $50 \mathrm{~mL}$ lactic acid, $30 \mathrm{~mL}$ nitric acid, and $2 \mathrm{~mL}$ hydrofluoric acid. The two phase $\gamma / \gamma$ ' microstructure was characterized using a high resolution FEI Sirion SEM. TEM foils were made using conventional preparation techniques. All foils were jet polished using a solution of $10 \%$ hydrochloric acid and $90 \%$ methanol at $-20^{\circ} \mathrm{C}$ and $13 \mathrm{~V}$. The substructures were characterized with an FEI CM200 TEM. Burgers vectors were determined by $\mathrm{g} \cdot \mathrm{b}$ analysis. Additionally, the thermally exposed but undeformed material obtained from the grip ends of a specimen was examined for comparison purposes.

\section{Results and Discussion}

\section{Fractography}

A comparison of the fracture surfaces in specimens with compressive and tensile dwells shows different failure mechanisms operating under each condition. Fractography indicated that specimens with compression hold cycles failed similarly at all temperatures surveyed. A representative fracture surface can be seen in Figure 3a, which has multiple crack initiation sites at the surface (A and B), with only one of the initiation sites (A) growing to form the main crack. Multiple surface initiation sites are common at high temperatures for coated specimens. Fatigue striations were observed, and a representative section of a fracture surface is shown in Figure $3 b$. The crack extension per cycle decreased during testing as would be expected in a total strain control test since the peak applied

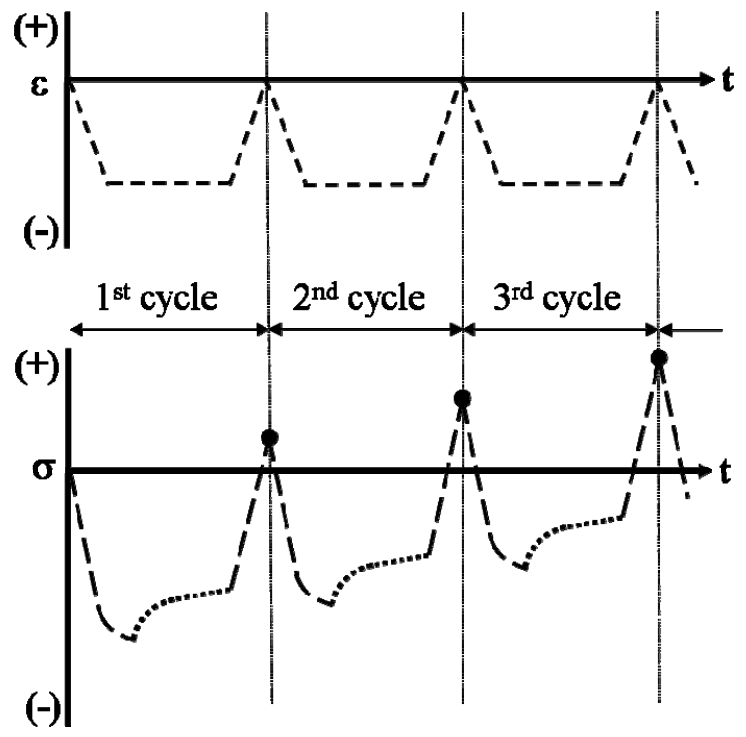

Figure 1. Schematic of applied strain during cyclic loading with compression hold time and the resulting stress response evolution with time.

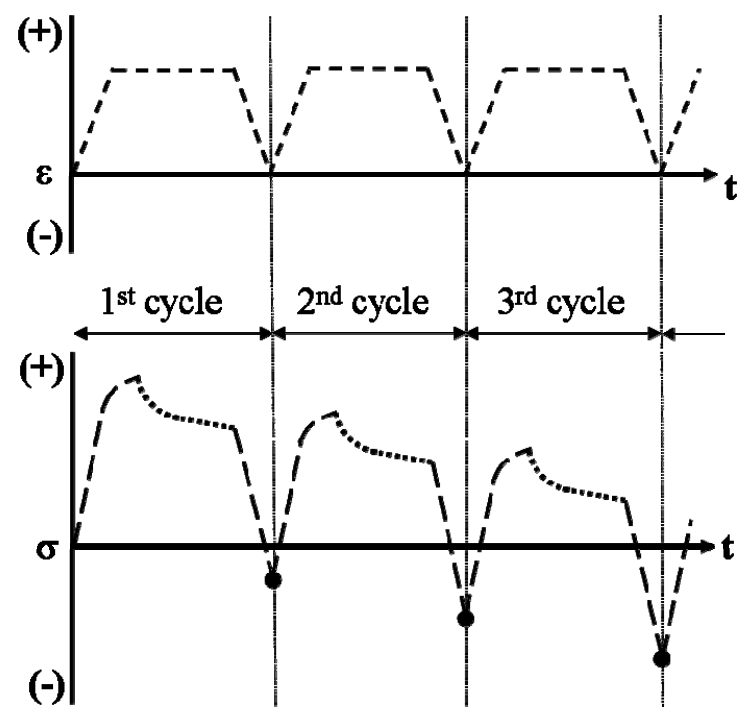

Figure 2. Schematic of applied strain during cyclic loading with tension hold time and the resulting stress response evolution with time.

stress intensity will decrease as the specimen becomes more compliant. The decrease in crack growth rate became more dramatic in specimens tested at higher temperatures. This was determined by the closer spacing of fatigue striations at longer crack lengths. The crack wake of each specimen was oxidized. Oxidation, which is prevalent at high temperatures, can increase crack closure and could be a contributing factor to the slowing crack propagation. Also, the plane of crack propagation changes from perpendicular to the loading direction during initiation to steep angles to the loading axis during propagation. Assuming the fatigue crack growth process is dominated by mode I loading, this 
transition from mode I to mixed mode loading may reduce the driving force for crack growth.

In the case of the tension hold, crack extension by creep deformation is observed in contrast to typical cyclic propagation. In this type of failure, seen in Figures $4 \mathrm{a}$ and $4 \mathrm{~b}$, there are flat, shallow dimples with evidence of casting pores. As these voids grow, they coalesce and the specimen finally fails. The fracture surfaces of the specimens were macroscopically flat compared to the compression hold specimens. In the tension dwell case, oxidation is seen over the entire surface, but does not appear to be as thick as the oxidation observed on the compression dwell tests. This oxidation most likely occurred when the samples were finally fractured at temperature, since ductile failure happens by the coalescence of voids that may not be exposed to the air during the SPLCF tests.

\section{Microstructure Characterization}

The $\gamma / \gamma^{\prime}$ microstructures in the SPLCF specimens with compression and tension dwell time were characterized and compared with the microstructure of the undeformed thermally exposed material. In the case of the thermally exposed material, cuboidal $\gamma^{\prime}$ dominated the structure, with no preferential

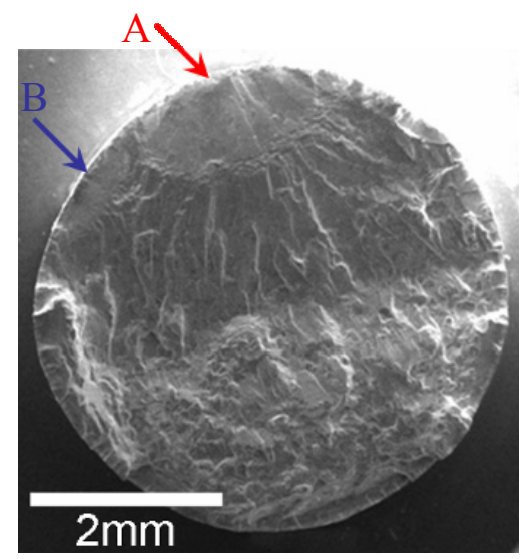

Figure 3a. Fracture surface of specimen with compression dwell. Arrows indicate surface initiation sites.

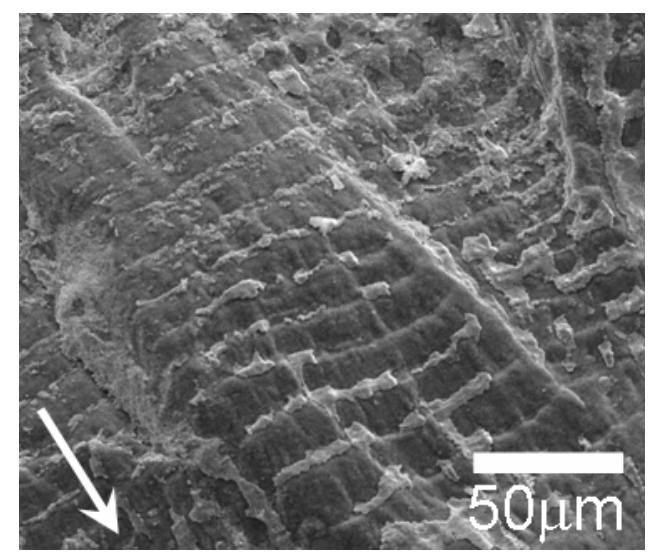

Figure 3b. Striations on the fracture surface of a compression dwell specimen. Arrow indicates direction of crack propagation. alignment of the $\gamma$ ' phase (i.e. rafting). The microstructure on the (001) and (100) crystallographic planes can be seen in Figures 5a and $5 \mathrm{~b}$, which are images taken parallel and perpendicular to the loading axis, respectively. In all figures, the $\gamma$ phase is a light gray while the $\gamma^{\prime}$ phase is a dark gray.

In the compression dwell tests, rafts formed parallel to the loading axis, referred to as "p-type" rafting. The flat sides of the rafts can be seen in Figure 6a. Characterization of this structure transverse to the loading axis shows that the rafts formed in orthogonal orientations, both parallel to the loading axis, seen in Figure $6 \mathrm{~b}$. In the case of the tension dwell tests, there was "n-type" rafting, or rafts perpendicular to the loading axis. Figure 7a shows the rafting parallel to the loading axis, while Figure $7 \mathrm{~b}$ shows the flat sides of the rafts, which are perpendicular to the loading direction. Both types of rafting are well documented for nickel-based superalloys with a negative $\gamma / \gamma^{\prime}$ misfit $[1,2,12,13]$.

In both tension and compression dwell specimens, the $\gamma^{\prime}$ phase became the continuous phase after loading, in contrast to the thermally exposed material where $\gamma$ is continuous. Additionally, a topological inversion has taken place, where the $\gamma^{\prime}$ phase has a larger area fraction after deformation. From the figures, it can be

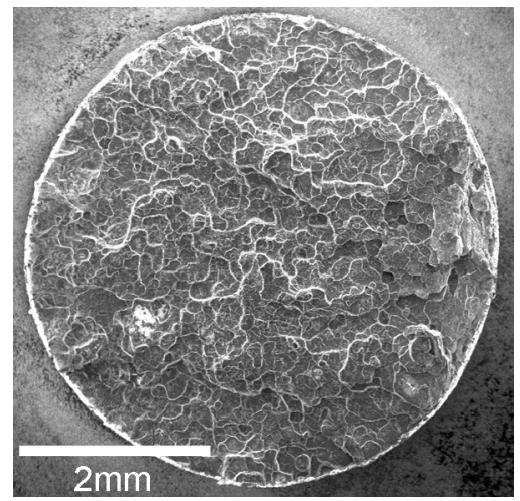

Figure 4a. Fracture surface of specimen with tension dwell. No obvious initiations sites at surface are observed.

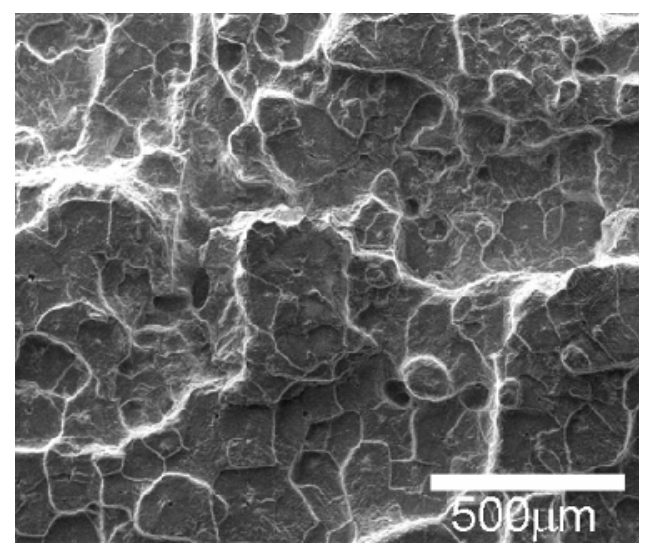

Figure 4b. Fracture surface of specimen with tension dwell. Shallow dimples can be seen, with casting pores throughout the surface. 
observed that the $\gamma$ phase is altered compared to specimens tested under creep alone. In creep, long continuous $\gamma$ and $\gamma^{\prime}$ channels are developed [2,5], but in the SPLCF specimens, the $\gamma$ phase has shorter sections, as seen in Figure 7a. Here the $\gamma$ channels are interrupted by the $\gamma^{\prime}$ phase coalescing with neighboring rafts. This is attributed to the creep-fatigue interaction, where the fatigue causes additional deformation that creep alone could not accomplish. Because interrupted tests were not done, it is not clear whether the rafts were ever long and continuous as expected during monotonic creep. It is known that rafts are caused by the superposition of the external stress and the coherency stress, which results in an unequal stress state developing in the $\gamma$ channels during deformation [1,2,14-16]. During tensile loading, the external stress reduces the compressive strain energy in the vertical channels relative to the horizontal channels. Stresses in the horizontal channels are relieved by the formation of dislocation networks. Because the stresses are not relieved in all directions, a gradient in elastic strain energy is formed between the horizontal and vertical channels. This gradient is the driving force for diffusional mass transport, and the rafted structure forms $[15,17]$. As seen in the schematics in Figures 1 and 2, the cyclic loading causes both compressive and tensile stresses in the specimen. Therefore the rafts do not fully develop because, in the case of cyclic fatigue, dislocations are forming in both horizontal and vertical channels, reducing the driving force for diffusional mass transport, and therefore rafting.

\section{$\underline{\text { Substructure Analysis }}$}

Even in the thermally exposed material, in the absence of applied stress, dislocations are present at $\gamma / \gamma^{\prime}$ interfaces, probably to accommodate the negative misfit of the $\gamma^{\prime}$ precipitates due to the rhenium additions [18]. Figure 8 shows that these dislocations travel through the $\gamma$ channels and loop around the $\gamma^{\prime}$ precipitates. The dislocations have burgers vectors of $\mathrm{a} / 2<110>$ and $\mathrm{a} / 2<\overline{1} 10>$. Intersecting dislocations form nodes and dislocation networks. No rafting was observed, however, after thermal exposure. In the absence of externally applied stresses, the dislocations move because of a combination of thermal gradient stresses and the stresses associated with the negative misfit of the $\gamma / \gamma$ ' interface. The dislocations achieve lower energy configuration by arranging into the network formation.

In the compression hold specimens, extensive dislocation networks formed in the $\gamma$ phase, as seen in Figure 9. Investigation of a large section of the sample revealed that dislocation networks formed in both vertical and horizontal $\gamma$ channels, along with $\gamma / \gamma^{\prime}$ interfaces. The dislocations in the networks have burgers vectors of $\mathrm{a} / 2<0 \overline{1} 1>, \mathrm{a} / 2<011>$, and $\mathrm{a} / 2<\overline{1} 10\rangle$. An isolated dislocation with a burgers vector of $\mathrm{a} / 2<0 \overline{1} 1>$ was found within the $\gamma^{\prime}$ phase that connected to the dislocations in the matrix networks. This indicates that the applied stress was large enough to cause dislocations to shear the $\gamma^{\prime}$ phase. While there were isolated dislocations shearing the $\gamma^{\prime}$ phase, most of the deformation appears to be confined to the $\gamma$ phase. Therefore, the dominant deformation mode was dislocation movement within the $\gamma$ channels and not by a $\gamma$ ' shearing mechanism.

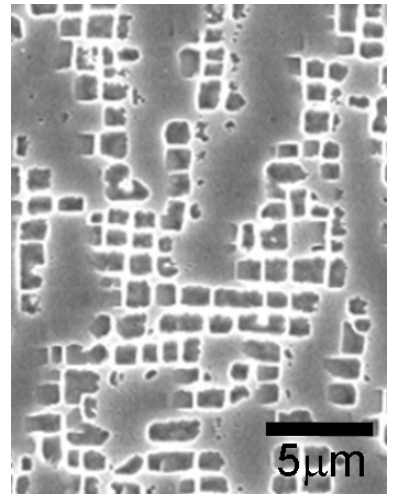

a)

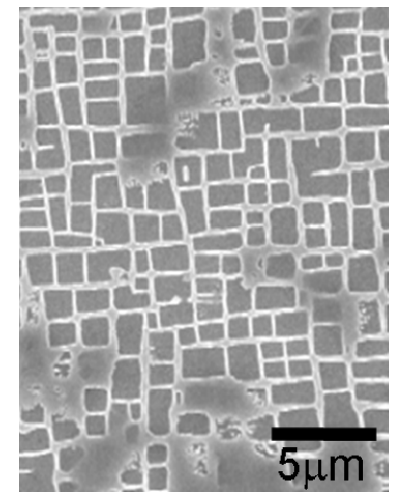

b)
Figure 5. High resolution SEM of the microstructure of thermally exposed material. No rafting is observed.

a) Parallel to [001]- specimen axis is vertical. b) Perpendicular to [100]- specimen axis is orthogonal to the image.

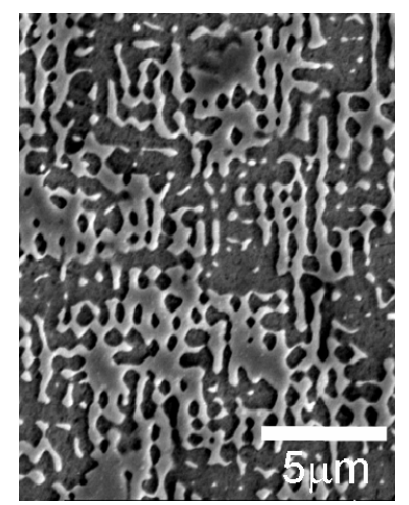

a)

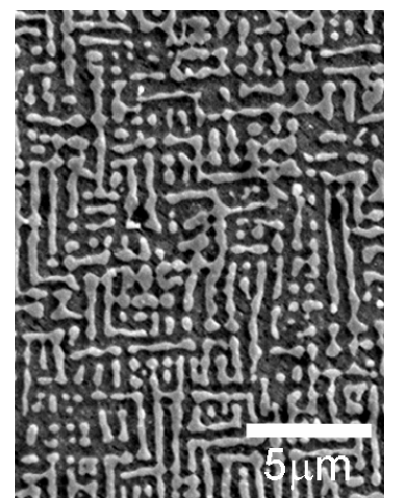

b)
Figure 6. Microstructure of specimen with compression dwell. Ptype rafting is observed. a) Parallel to load- [100] normal, loading axis is vertical. b) Perpendicular to load- [001] normal- specimen axis is orthogonal to image.

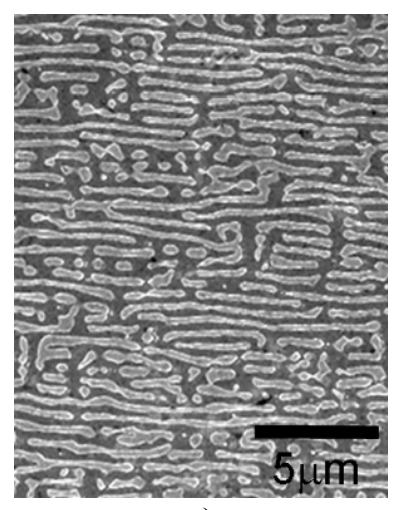

a)

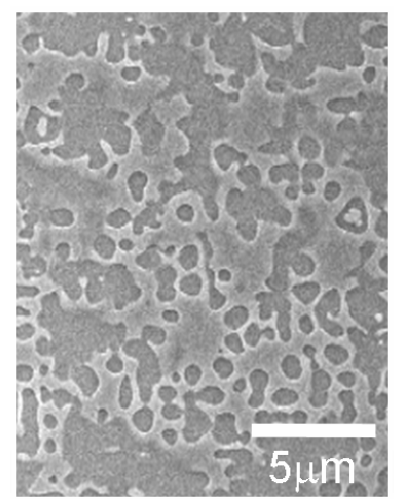

b)
Figure 7. Microstructure of specimen with tension dwell. N-type rafting is observed. a) Parallel to load- [100] normal, loading axis is vertical. b) Perpendicular to load- [001] normal- specimen axis orthogonal to image. 


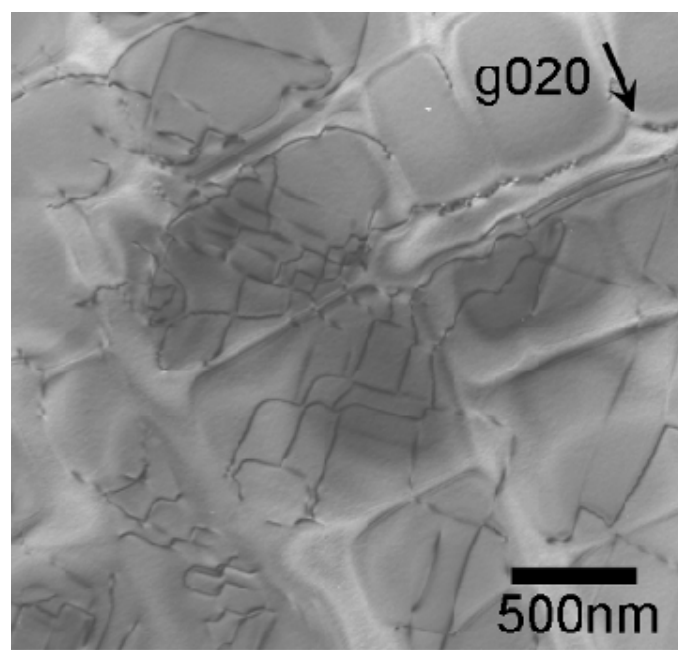

Figure 8. Bright field TEM image of the thermally exposed material. Dislocation networks form at $\gamma / \gamma^{\prime}$ interfaces to accommodate the negative misfit. Loading axis is normal to image.

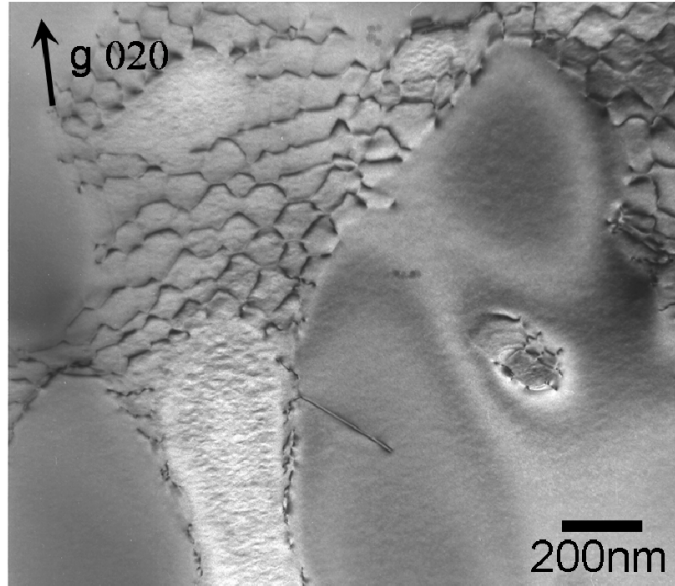

Figure 9. Bright field TEM image of specimen with compression dwell. Dislocation motion in the $\gamma$ channels dominates the deformation. Loading axis is nearly horizontal.

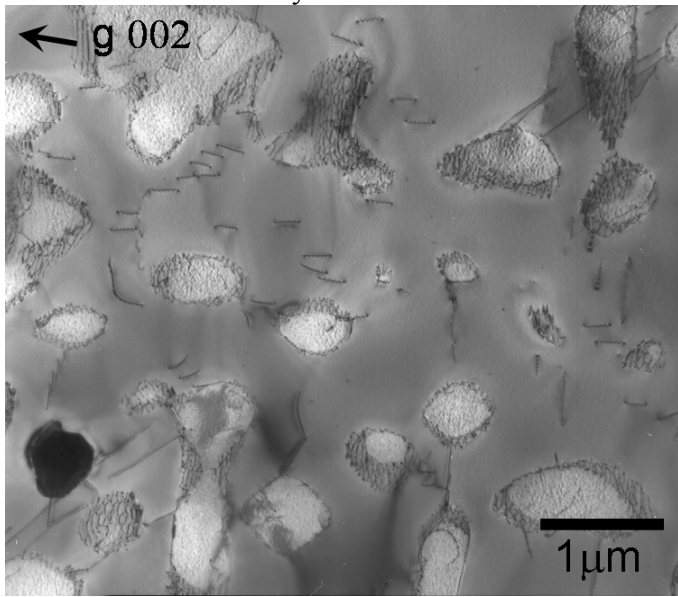

Figure 10. Bright field TEM image of specimen with tension dwell. Shearing of $\gamma^{\prime}$ particles was the primary deformation mode. Loading axis is nearly horizontal.
In the tension hold specimens, the dislocation networks were confined to the $\gamma / \gamma^{\prime}$ interfaces, shown in Figure 10. The dislocation networks were again seen in both horizontal and vertical channels. Multiple dislocations with burgers vectors of $<101>$ were observed in the $\gamma^{\prime}$ phase. Screw dislocations were observed shearing through multiple $\gamma^{\prime}$ particles at once, while others were bowing within the $\gamma^{\prime}$ phase. Since the dislocation networks were confined to the interface, the $\gamma^{\prime}$ appears to be an important deformation mode, suggesting that at least at this stage of deformation, the $\gamma$ ' phase is acting as the continuous "matrix" phase.

TEM observations from both samples reveal that dislocation networks have formed in both vertical and horizontal $\gamma$ channels and along all $\gamma / \gamma^{\prime}$ interfaces. As stated earlier, the formation of dislocations along both types of channels decreases the driving energy for diffusional mass transport. Therefore it is evident through the TEM studies that the cyclic loading is deterring raft formation.

\section{Conclusions}

In this work, the microstructural changes occurring in SPLCF specimens with tension and compression dwell times were compared with thermally exposed material. It was observed that the combination of creep and fatigue loading caused short, discontinuous rafts, in comparison to rafts observed in monotonic creep. TEM analysis confirmed that dislocations were moving in both horizontal and vertical channels, and therefore deterring raft formation because the driving force for diffusional mass transport is being reduced by the strain accommodation. The reduced driving force does not allow long continuous rafts to form as easily, and the short, discontinuous rafting was observed.

Dislocation network formation and $\gamma$ ' shearing appears to be the main deformation mode for compression dwell and tension dwell, respectively. For each of the SPLCF specimens it is difficult to distinguish what deformation is due to monotonic loading or cyclic loading. This highlights the need for a separate fatigue study, where the fatigue mechanisms can be described before attempting to analyze the combined, and probably synergistic, effect of creep and fatigue.

\section{Acknowledgements}

This work was supported by the Air Force Office of Scientific Research under award No. FA9550-05-1-0135.

\section{References}

1. Mughrabi, H. and U. Tetzlaff, "Microstructure and HighTemperature Strength of Monocrystalline Nickel-Base Superalloys," Advanced Engineering Materials, 2 (6)(2000), 319326.

2. Kamaraj, M., "Rafting in single crystal nickel-base superalloys- An overview," Sadhana, 28 (1\&2)(2003), 115-128.

3. Epishin, A., T. Link, et al., "Evolution of the $\gamma / \gamma^{\prime}$ microstructure during high temperature creep of a nickel-base superalloy," Acta Materialia, 48 (2000), 4169-4177. 
4. Epishin, A., T. Link, et al., "Influence of high temperature creep damage on low cycle fatigue of CMSX-4" (Paper presented at Materials Science \& Technology 2005, Pittsburgh, PA, 2005), 231-239.

5. Reed, R. C., N. Matan, et al., "Creep of CMSX-4 superalloy singel crystals: Effects of rafting at high temperature," Acta Materialia, 47 (12)(1999), 3367-3381.

6. Legros, M., N. Clement, et al., "In-situ observation of deforation micromechanisms in a rafted $\gamma / \gamma^{\prime}$ superalloy at $850 \mathrm{C}$," Materials Science and Engineering A, 337 (2002), 160-169.

7. Li, J. R., Z. G. Zhong, et al., "A low-cost second generation single crystal superalloy DD6" (Paper presented at Superalloys 2000, Champion, PA, 2000), 777-783.

8. Zheng, Y., X. Wang, et al., "Effect of Ru Addition on Cast Nickel Base Superalloy with Low Content of Cr and High Content of W" (Paper presented at Superalloys 2000, Champion, PA, 2000), 305-311.

9. Zhou, H., Y. Ro, et al., "Deformation Microstructures after low-cycle fatigue in a fourth-generation Ni-based SC superalloy TMS-138," Materials Science and Engineering A, 381 (2004), 2027.

10. Zrnik, J., J. A. Wang, et al., "Influence of cycling frequency on cyclic creep characteristics of nickel base single-crystal superalloy," Materials Science and Engineering A, 234-236 (1997), 884-888.

11. Zrnik, J., Z. G. Wang, et al., "High Temperature Creep/Fatigue Deformation of Single Crystal Nickel Base Superalloy" (Paper presented at 10th International Conference on the Strength of Materials and Alloys, Sendai, Japan, 1994), 587590.

12. Ratel, N., G. Bruno, et al., "Plastic strain-induced rafting of $\gamma^{\prime}$ precipitates in Ni superalloys: Elasticity analysis," Acta Materialia, 54 (2006), 5087-5093.

13. Tian, S., X. Yu, et al., "Deformation features of a nickel-base superalloy single crystal during compression creep," Materials Science and Engineering A, 379 (2004), 141-147.

14. Reed, R. C., The Superalloys: Fundamentals and Applications.(New York: Cambridge University Press, 2006) 180187.

15. Mughrabi, H., M. Ott, et al., "New microstructural concepts to optimize the high-temperature strength of $\gamma^{\prime}$-hardened monocrystalline nickel-based superalloys," Materials Science and Engineering A, 234-236 (1997), 434-437.

16. Pineau, A., "Influence of uniaxial stress on the morphology of coherent precipitates during coarsening- Elastic energy considerations," Acta Metallurgica, 24 (1976), 559-564.
17. Mishin, Y., "Atomistic modeling of $\gamma$ and $\gamma^{\prime}$-phases of the NiAl system," Acta Materialia, 52 (2004), 1451-1467.

18. Giamei, A. F. and D. L. Anton, "Rhenium Additions to a Nibase Superalloy: Effects on Microstructure," Materials Science and Engineering A, 16A (1985), 1997-2005. 